\title{
Esferas (Beads) de Alginato como Agente Encapsulante de Óleo de Croton Zehntneri Pax et Hoffm
}

\author{
Haroldo C. B. de Paula, Erick F. de Oliveira, Flavia 0. M. S. Abreu \\ Departamento de Química Analítica e Físico-Química, UFC
}

\author{
Regina C. M. de Paula \\ Departamento de Química Orgânica e Inorgânica, UFC
}

\author{
Selene M. de Morais \\ Laboratório de Produtos Naturais, UECE
}

\author{
Maria M. C. Forte \\ Laboratório de Materiais Poliméricos, UFRGS
}

\begin{abstract}
Resumo: Esferas de alginato (ALG) reticuladas com cálcio e revestidas com goma do cajueiro (GC) foram preparadas e dopadas com óleo essencial de Croton zehntneri Pax et Hoffm $(\mathrm{Cz})$, visando a seu emprego como larvicida para combate ao vetor da dengue. As esferas foram caracterizadas estruturalmente por espectroscopia de infravermelho, análise térmica, microscopia eletrônica de varredura e em relação aos seus parâmetros de transporte líquido, dopagem, embebição $(\mathrm{Q})$ e cinética de liberação in vitro e in vivo. Os resultados revelaram que as esferas possuem morfologia com uma alta regularidade esférica, com superfície porosa. A matriz ALG:Cz =1:1 apresentou maior dopagem e eficiência de encapsulamento e valores de Q entre 10 e 12. O perfil de liberação do óleo encapsulado apresentou-se mais prolongado nas esferas contendo goma do cajueiro, ALG-GC:Cz =1:1. A taxa de mortalidade de larvas de Stegomyia aegypti para teores de 1,81 a 4,25 mg de óleo nas esferas resultou em valores na faixa de 72 a 100\%, após 24 h. A matriz polimérica mostrou-se efetiva para proteção do principio ativo até cerca de 70 dias, com perdas da ordem de 35\%. A análise térmica revelou que a incorporação de Cz e GC resulta em uma matriz polimérica termicamente mais estável.
\end{abstract}

Palavras-chave: Alginato, esferas, goma do cajueiro, encapsulação, Stegomyia aegypti.

\section{ALG/Ca Beads as an Encapsulation Agent of Croton Zehntneri Pax et Hoffm Essential Oil}

\begin{abstract}
Alginate beads (ALG) crosslinked with calcium and coated with cashew gum (GC) were prepared and loaded with an essential oil from Croton zehntneri Pax et $\operatorname{Hoffm}(\mathrm{Cz})$. The beads were characterized by FTIR, thermal properties, with respect to their size, shape, larvicide loading, swelling, and in vitro and in vivo release kinetics. Results showed that beads with ALG: $\mathrm{Cz}=1: 1$ relative ratio showed higher loading and encapsulation efficiency, although presenting a faster release kinetics. On the other hand, ALG beads coated with GC showed Q values from 10 to 12, representing a significant increase in the hydrophilic character of the matrix. Infrared spectroscopy and thermal analysis confirmed the presence of essential oil encapsulated in beads. Morphologic investigation by SEM revealed a highly spherical and porous surface. The in vitro profile showed that the $\mathrm{Cz}$ oil encapsulated had a prolonged release in ALG-GC beads, probably due to the crosslinking. In vivo tests showed that the mortality degree was related to the mass of the beads, where beads having oil content from 1.81 to $4.25 \mathrm{mg}$ caused larvae mortalities from 72 to $100 \%$ after $24 \mathrm{~h}$. The control release system was found to be effective up to 70 days. These results showed that ALG-GC beads loaded with $\mathrm{Cz}$ are promising candidate for the control of Stegomyia aegypti larvae.
\end{abstract}

Keywords: Alginate, spheres, cashew gum, encapsulation, Stegomyia aegypti.

\section{Introdução}

Vários sistemas à base de biopolímeros têm sido usados como matrizes para liberação prolongada de fármacos, princípios ativos e inseticidas. Tais sistemas são geralmente biocompatíveis, biodegradáveis e oriundos de matérias primas naturais, o que torna estes materiais de especial interesse para diversas áreas de pesquisa ${ }^{[1-6]}$. Microesferas são um exemplo de tais sistemas que têm despertado bastante interesse por parte da academia, pois podem ser usados para a liberação prolongada de vários princípios ativos empregados nas áreas farmacêutica, médica e da agricultura ${ }^{[1-10]}$.

Autor para correspondência: Haroldo C. B. de Paula, Departamento de Química Analítica e Físico-Química, Universidade Federal do Ceará, Campus do Pici, CEP: 60451-970, Fortaleza, CE, Brasil.E-mail: hpaula@ufc.br 
O alginato (ALG) é um biopolímero aniônico composto de cadeias lineares de ácido $\alpha$-L-glucurônico e $\beta$-D-mannurônico, as quais em presença de íons tais como $\mathrm{Ca}^{++}$formam hidrogéis, filmes, esferas, micro- e nanopartículas, com capacidade para encapsulamento de uma série de substâncias tais como dexorubicina, insulina, antifúngicos, etc. ${ }^{[2,4,11-13]}$. Os métodos de preparação geralmente envolvem o controle do processo de gelificação, o qual é função da concentração do polímero e do contra-íon, da velocidade de adição da solução de contra-íon, etc. Matrizes de ALG/Ca (microesferas) têm sido também empregadas para a liberação de inseticidas ${ }^{[13,14]}$. Géis contendo biopolímeros regionais tais como goma do cajueiro e quitosana foram preparado ${ }^{[15]}$ tendo sido usados para encapsulamento de inseticidas convencionais ${ }^{[16]}$ e para a liberação de drogas ${ }^{[17]}$.

A goma de cajueiro (GC) é um polissacarídeo oriundo da Anacardium occidentale (árvore do cajueiro), sendo constituída de galactose (72\%), D-glicose (14\%), arabinose $(4,6 \%)$, rhamnose $(3,2 \%)$ e ácido glucurônico $(4,7 \%)^{[18]}$. A goma do cajueiro possui propriedades semelhantes à goma arábica, podendo ser empregada como agente encapsulante em sistemas de liberação de princípios ativos com aplicações variadas nas indústrias de alimentos, médica, farmacêutica e na agricultura.

Croton zehntneri Pax et Hoffm, é um arbusto do Nordeste brasileiro, sendo popularmente conhecido como "canela de cunhã", apresentando em seus talos e folhas óleo essencial cujo principal componente (cerca de 47\%) é o anetol $\left(\mathrm{C}_{10} \mathrm{H}_{12} \mathrm{O}\right)$. O potencial antioxidante ${ }^{[19]}$ anti-helmíntico ${ }^{[20]}$ e larvicida ${ }^{[21]}$ deste óleo tem sido investigado, tendo sido reportado neste ultimo caso um valor de $\mathrm{LC}_{50}$ de $28 \mathrm{ppm}$. A volatilidade e a degradabilidade do seu óleo essencial requer o desenvolvimento de metodologias de encapsulamento que visem preservar o seu princípio ativo, além de facilitar o manuseio dos eventuais produtos finais.

As epidemias de dengue que continuam a assolar o País clamam pela busca contínua de pesquisas que visem contribuir de forma efetiva para o controle do vetor de infestação. Neste sentido, pesquisas que objetivem a eliminação das larvas do Stegomyia aegypti usando substâncias com impacto mínimo ou nulo sobre o meio ambiente têm sido realizadas empregando géis e microesferas para encapsulamento de vários biolarvicidas ${ }^{[22,23]}$.

Este trabalho teve como finalidade o desenvolvimento de esferas de alginato e goma do cajueiro dopadas com óleo essencial de Croton zenhtneri $(\mathrm{Cz})$, com atividade larvicida, com o objetivo de preservar o principio ativo e prolongar a liberação do mesmo no meio. Investigou-se ainda o efeito da adição de goma de cajueiro (GC) nas propriedades físico-químicas das esferas, realizando-se estudos de cinética de liberação in vitro e bioensaios de mortalidade com larvas de Stegomyia aegypti.

\section{Experimental}

\section{Reagentes}

Alginato sódico de média viscosidade $(\geq 2,000 \mathrm{cP}$, SIGMA), cloreto de cálcio (SYNTH), Tween 80 (SYNTH) foram usados sem nenhuma purificação prévia; o óleo das folhas de canela-de-cunhã foi obtido por hidrodestilação em um aparelho de Clevenger e cedido pelo Laboratório de Produtos Naturais da UECE. A goma de cajueiro $\left(1,1 \times 10^{5} \mathrm{~g} \cdot \mathrm{mol}^{-1}\right)$ foi obtida da Anacardium Occidentale e purificada conforme descrito em trabalho anterior ${ }^{[18]}$.

\section{Preparação das esferas de alginato}

À uma solução aquosa de alginato (ALG) na concentração de $1,5 \%\left(\mathrm{~m} . \mathrm{v}^{-1}\right)(0,23$ mmolar $), 2,5 \%(0,38 \mathrm{mmolar})$ ou $5 \%$ (0,78 mmolar) foram adicionadas glicerina, surfactante (Tween 80) e óleo essencial (Cz) em diferentes proporções $(\mathrm{ALG} / \mathrm{Cz})=1: 1,2: 1,1: 2,3: 1$. A mistura foi colocada em um emulsificador (PHOENIX, modelo AP-56) por 15 minutos, à temperatura ambiente, para estabilização da emulsão, a qual foi a seguir gotejada em uma solução de $\mathrm{CaCl}_{2} 15 \%$ (1 M), através de uma seringa ( $22 \mathrm{G}$ ) usando uma bomba peristáltica com fluxo de $1,6 \mathrm{~mL} / \mathrm{min}$. As esferas foram deixadas em repouso por 5 minutos, e posteriormente foram removidas, lavadas com água destilada e secadas em dessecador por 48 horas. Cada formulação foi realizada em duplicata.

\section{Preparação das esferas de ALG revestidas com GC (ALG-GC)}

As esferas de ALG revestidas com GC foram preparadas conforme descrito anteriormente, contudo, após as esferas serem removidas da solução de $\mathrm{CaCl}_{2}$, foram imersas em solução de $10 \%$ GC (10 mmolar) por 30 minutos e posteriormente em solução de glutaraldeido $50 \%$, onde permaneceram sob agitação por 30 minutos a $50{ }^{\circ} \mathrm{C}$. As esferas foram então removidas, lavadas com água destilada e secas em dessecador durante 48 horas.

\section{Dopagem das esferas e eficiência de encapsulamento}

O teor de óleo presente nas esferas foi determinado em triplicata por através de espectroscopia na região do UV-Vis, no comprimento de onda de $259 \mathrm{~nm}$. Macerou-se cerca de $5 \mathrm{mg}$ de esferas em etanol, e calculou-se a concentração de óleo através de uma curva de calibração dada pela Equação 1:

$$
\text { Abs }=-0,010+0,057 . \text { Conc. } \quad R^{2}=0,9994
$$

onde $A b s$ representa absorbância e Conc a concentração de óleo em ppm.

A dopagem das esferas foi calculada em percentual a partir da concentração de óleo em ppm (Conc), do volume da solução em etanol (Vsol) e a massa de amostra ( $\left.\mathrm{m}_{\text {amostra }}\right)$, conforme a Equação 2.

$$
\operatorname{Dopagem}(\%)=\frac{\text { Conc. } \cdot \text { sol.100 }}{1000 \cdot \mathrm{m}_{\text {amostra }}}
$$

A eficiência de encapsulamento (EE) foi calculada através da razão entre o percentual de óleo efetivamente encapsulado nas esferas e o percentual relativo de óleo adicionado no processo, sendo expressa em porcentagem.

\section{Embebição e transporte líquido}

A embebição (Q) foi realizada pesando-se em uma balança de precisão cerca de $10 \mathrm{mg}$ de esferas secas, dopada e não-dopada e colocando-as em um recipiente com água 
destilada. Após um certo intervalo de tempo, as esferas foram retiradas do meio, o excesso de água foi removido colocando-as em um fino papel absorvente e posteriormente as esferas embebidas foram pesadas em certos intervalos de tempo. Q foi obtida de acordo com a Equação $3^{[3,9]}$ :

$$
\mathrm{Q}=\frac{\left(\mathrm{M}_{\mathrm{t}}-\mathrm{M}_{0}\right)}{\mathrm{M}_{0}}
$$

onde $\mathrm{M}_{\mathrm{o}}$ é massa das esferas secas e $\mathrm{M}_{\mathrm{t}}$ depois de imersas em água em um dado intervalo de tempo.

\section{Estudo cinético da liberação de óleo in vitro}

Uma determinada massa de esferas dopadas (50 $\mathrm{mg}$ ) foi colocada em uma célula em um béquer com $120 \mathrm{~mL}$ de água destilada. O sistema foi mantido na temperatura de $20{ }^{\circ} \mathrm{C}$, sob agitação. Após certos intervalos de tempo retirava-se uma alíquota e analisava-se por espectroscopia na região do UV-Vis calculando-se a concentração de óleo presente através da curva de calibração mencionada na Equação 1.

\section{Taxa de mortalidade larval}

A liberação in vivo de esferas dopadas com Cz foi realizada a fim de determinar a mortalidade de larvas do St. aegypti. Para isto, determinada massa de esferas $(10,20,30 \mathrm{ou} 50 \mathrm{mg})$ foi colocada em $50 \mathrm{~mL}$ de água destilada, contendo uma população de vinte larvas de St. Aegypti, no estágio três (classe Rockerfeller). A população de larvas mortas foi determinada após 24 e 48 horas de exposição. Cada teste foi realizado em triplicata, utilizando uma amostra com esferas não-dopadas, como controle ${ }^{[24]}$.

\section{Análises e caracterização}

Determinou-se o diâmetro das esferas com um micrômetro Mitutoyo e a massa das esferas através de pesagem em balança analítica de precisão (Micronal). As esferas foram ainda caracterizadas por microscopia eletrônica de varredura em um equipamento Jeol JSM 5800 usando uma aceleração de voltagem de $10 \mathrm{kV}$ e por espectroscopia na região do infravermelho com transformada de Fourier (FTIR) usando pastilhas de KBR, em um aparelho da Perkin Elmer.

\section{Analise térmica}

A estabilidade térmicas das esferas foi avaliada por analise termogravimétrica (TGA) em um equipamento Shimadzu modelo TGA 50 usando uma rampa de aquecimento de $10{ }^{\circ} \mathrm{C} /$ min entre 25 a $600{ }^{\circ} \mathrm{C}$, e por Calorimetria de Varredura Diferencial (DSC) em um equipamento Shimadzu modelo DSC 50 aquecendo-se cerca de $5 \mathrm{mg}$ de 25 a $300{ }^{\circ} \mathrm{C}$ usando uma taxa de aquecimento de $10{ }^{\circ} \mathrm{C} / \mathrm{min}$.

\section{Resultados e Discussão}

\section{Caracterização das esferas}

Foram preparadas esferas partindo-se de solução de ALG de diferentes concentrações, mantendo-se a razão relativa fixa em 1:1 ALG:Cz $(\mathrm{m} / \mathrm{m})$, com o objetivo de observar o efeito da concentração de ALG na dopagem e no diâmetro médio das esferas. A Tabela 1 sumariza os dados obtidos.

As esferas de ALG dopadas com $\mathrm{Cz}$ apresentaram um diâmetro médio que variou de 0,71 para $1,46 \mathrm{~mm}$, para faixas de concentração de ALG estudada. As esferas apresentaram valores médios de dopagem de 1,4 a $8,7 \%$, em função da concentração inicial de ALG. A dopagem foi maximizada quando as esferas foram formuladas com 2,5\% ALG, apresentando um valor máximo médio de $8,7 \%$, com mais baixo desvio padrão. A massa média das esferas foi maior para a concentração de 2,5\% ALG, provavelmente devido a maior dopagem. A partir deste resultado, novas formulações foram desenvolvidas, buscando-se observar o efeito da adição de GC e variando-se o teor relativo $\mathrm{ALG} / \mathrm{Cz}$ na matriz. Os dados obtidos podem ser vistos na Tabela 2 . Verifica-se que a dopagem das esferas variou de 2,6 a $10,7 \%$, cujo aumento foi proporcional a quantidade de $\mathrm{Cz}$ na formulação. A eficiência de encapsulamento foi maximizada nas formulações ALG:Cz 1:1 e ALG-GC:Cz 1:1, apresentando valores de 26 e $25 \%$. Os valores de dopagem estão de acordo com os encontrados em trabalhos correlatos sobre encapsulamento de pesticidas em esferas e microesferas. El Bahri e colaboradores $^{[25]}$ encapsularam um herbicida comercial em microesferas de derivados de celulose, cujos valores de dopagem variaram de $8-11 \%$, enquanto que esferas de alginato dopadas com Neem (Azadirachta Indica A. Juss), um pesticida natural, apresentaram valores de dopagem na faixa de 10 a $30 \%$ [12].

A Figura 1 mostra micrografias das esferas de ALG não-dopada e dopada com Cz (ALG:Cz 1:1). A Figura 1 mostra que as esferas de ALG apresentaram morfologia esferóide, no qual a esfera ALG:Cz 1:1 (Figura 1b) apresenta maior regularidade esférica do que a esfera não dopada (Figura 1a).

Tabela 1. Massa, diâmetro médio e dopagem das esferas em função da concentração de ALG.

\begin{tabular}{cccc}
\hline $\begin{array}{c}\text { Concentração de } \\
\text { Alginato }(\%)\end{array}$ & $\begin{array}{c}\text { Massa } \\
(\mathbf{m g})\end{array}$ & $\begin{array}{c}\text { Diâmetro } \\
(\mathbf{m m})\end{array}$ & $\begin{array}{c}\text { Dopagem } \\
(\mathbf{\%})\end{array}$ \\
\hline $1,5 \%$ & $0,7 \pm 0,2$ & $0,71 \pm 0,1$ & $3,7 \pm 1$ \\
$2,5 \%$ & $1,9 \pm 0,5$ & $1,04 \pm 0,1$ & $8,7 \pm 0,7$ \\
$5 \%$ & $1,3 \pm 0,3$ & $1,46 \pm 0,1$ & $1,4 \pm 0,2$ \\
\hline
\end{tabular}

Tabela 2. Dopagem e eficiência de encapsulamento (EE) para diferentes esferas.

\begin{tabular}{lccc}
\hline \multicolumn{1}{c}{ Formulação } & $\begin{array}{c}\text { Razão } \\
\text { ALG/Cz }\end{array}$ & $\begin{array}{c}\text { Dopagem } \\
(\boldsymbol{\%})\end{array}$ & $\begin{array}{c}\text { EE } \\
(\boldsymbol{\%})\end{array}$ \\
\hline ALG:Cz 1:2 & $1: 2$ & $10,7 \pm 0,5$ & 26 \\
ALG:Cz 1:1 & $1: 1$ & $8,7 \pm 0,7$ & 26 \\
ALG-GC:Cz 1:1 & $1: 1$ & $8,2 \pm 0,2$ & 25 \\
ALG:Cz 4:3 & $4: 3$ & $5,1 \pm 0,3$ & 17 \\
ALG:Cz 2:1 & $2: 1$ & $3,2 \pm 0,5$ & 13 \\
ALG-GC:Cz 2:1 & $2: 1$ & $2,6 \pm 0,1$ & 15 \\
ALG:Cz 3:1 & $3: 1$ & $1,2 \pm 0,4$ & 6 \\
\hline
\end{tabular}


Micrografias com maior magnificação das esferas não dopadas e dopadas (Figuras 1c e d, respectivamente) revelam que a superfície da esfera não dopada é altamente porosa, enquanto que a esfera de ALG dopada com Cz apresenta uma superfície mais homogênea e lisa devido à presença de óleo. Estes resultados estão de acordo com o observado por Tu et al. ${ }^{[6]}$, no qual a morfologia das micropartículas de ALG-Ca revelou-se irregular e de superfície porosa. Kulkarni e colaboradores, por outro lado, reportaram em diversos trabalhos o encapsulamento de cefadroxil ${ }^{[9]}$, neem ${ }^{[12]}$ e clorpirofós ${ }^{[1]}$ em esferas de ALG-Ca as quais exibiram uma morfologia esferóide, porém com superfície lisa e regular.

As esferas de ALG dopadas com $\mathrm{Cz}$ e não-dopadas, bem como aquelas revestidas com GC foram caracterizadas através da análise de seus principais grupamentos funcionais, por espectroscopia de infravermelho (FT-IR), conforme ilustrado na Figura 2.

A análise espectrofotométrica das esferas de ALG não dopada e dopada revelou a presença de sinais principais característicos do ALG em $1652 \mathrm{~cm}^{-1}$ atribuído ao estiramento -COO- em $1097 \mathrm{~cm}^{-1}$ devido ao estiramento -C-O-C-; e em $3442 \mathrm{~cm}^{-1}$ atribuído ao estiramento do grupamento hidroxila e por interações do tipo pontes de hidrogênio ${ }^{[3,26]}$. As esferas de ALG quando revestidas com GC

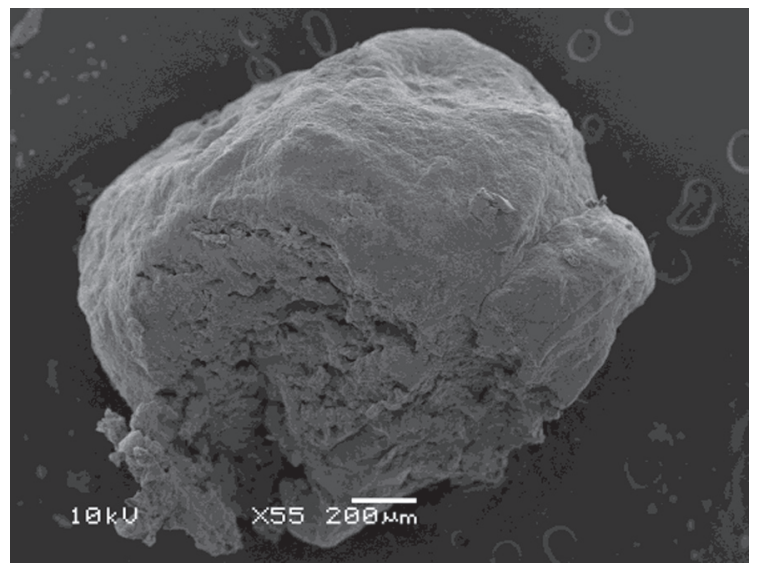

(a)

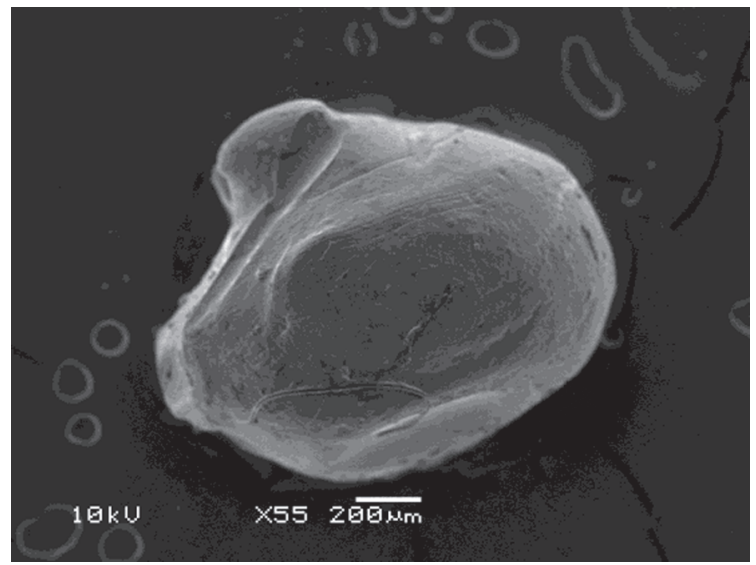

(b) apresentam o deslocamento do estiramento do grupamento -COO- de 1652 para $1638 \mathrm{~cm}^{-1}$ devido a introdução de novos grupamentos carboxílicos, e novos sinais em torno de $1700 \mathrm{~cm}^{-1}$ devido a reticulação de suas cadeias. $\mathrm{O} \mathrm{Cz}$ apresenta os principais sinais característicos referentes ao anetol em $3000 \mathrm{~cm}^{-1}$ da ligação $\mathrm{O}-\mathrm{CH}_{3}$ e as vibrações $\mathrm{C}-\mathrm{H}$ do anel aromático em 1460, 1518 e $1612 \mathrm{~cm}^{-1[27]}$. As esferas de ALG e de ALG-GC dopadas com Cz apresentaram o sinal característico do $\mathrm{Cz}$ em $1460 \mathrm{~cm}^{-1}$, além dos deslocamentos das bandas da hidroxila e dos carbonos C-H. Os demais sinais característicos do $\mathrm{Cz}$ ficaram sobrepostos aos sinais dos grupamentos polissacarídicos da matriz.

\section{Embebição e transporte líquido}

O grau de embebição avalia a capacidade de hidratação das esferas com o tempo até o equilíbrio. A embebição é uma etapa importante no processo de difusão de um soluto em uma matriz polimérica, daí deve-se conhecer o coeficiente de difusão $D_{v}$, o qual pode ser obtido a partir da Equação $4^{[28]}$ :

$$
\mathrm{D}_{\mathrm{v}}=\left(\frac{\mathrm{r} \theta}{6}\right)^{2} \Pi
$$

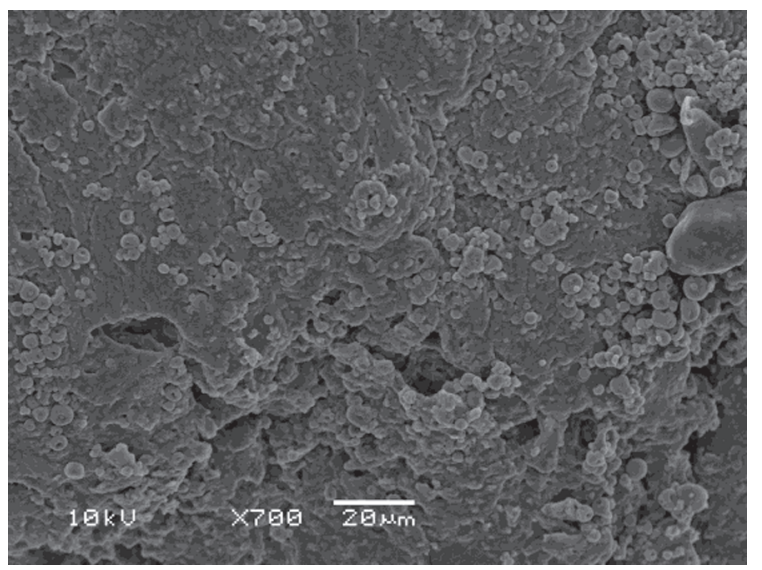

(c)

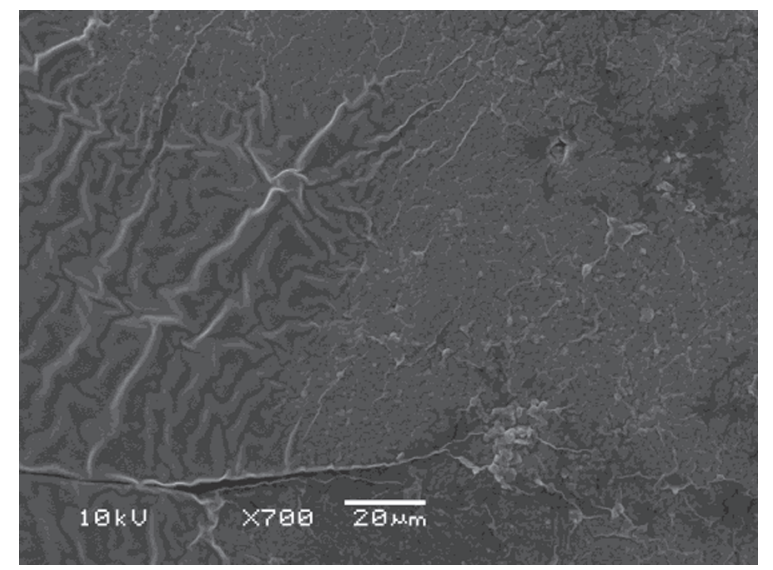

(d)

Figura 1. Micrografia das esferas de ALG não dopadas a) e dopadas com Cz b); Magnificação da superfície (700×) das esferas não dopadas c) e dopadas com Cz d). 
onde $\mathrm{D}_{\mathrm{v}}$ é o coeficiente de difusão, $\mathrm{r}$ o raio da esferas, $\theta$ a inclinação da região linear do gráfico de $\ln \left(\mathrm{M}_{\mathrm{t}} / \mathrm{M}_{\infty}\right)$ versus $V_{t} . M_{t}$ é a massa de água embebida no tempo t, $M_{\infty}$ a massa embebida no equilíbrio. Os dados calculados são mostrados na Tabela 3.

As esferas de ALG apresentaram os valores de embebição (Q) no equilíbrio de 0,6 para as esferas não-dopadas e 0,86 para as esferas dopadas, enquanto que as esferas de ALG-GC apresentaram valores de 10,6 para as esferas não-dopadas e de 12,2 para as esferas dopadas. O coeficiente de difusão aumentou significativamente com a incorporação de $\mathrm{Cz}$ nas

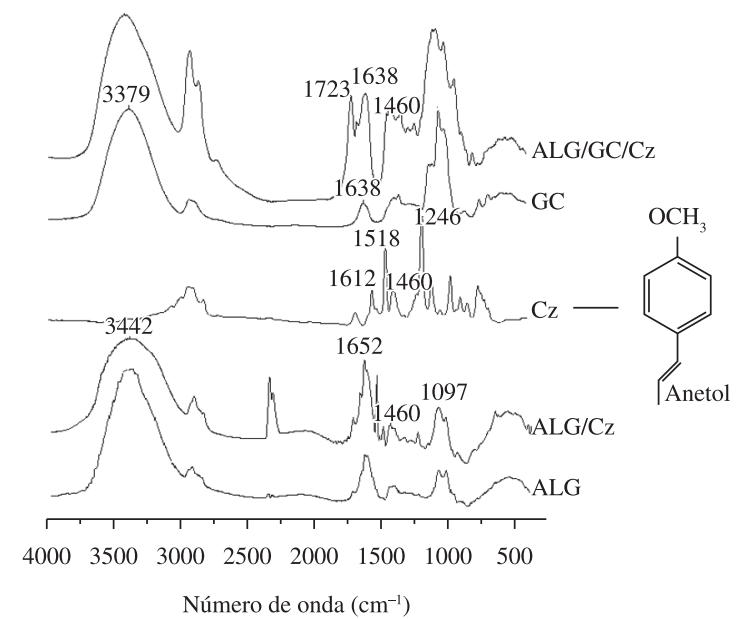

Figura 2. FT-IR da Goma de Cajueiro (GC), do Cz e das esferas de ALG não dopadas e dopadas com $\mathrm{Cz}$ e das esferas ALG-GC dopadas com Cz.

Tabela 3. Parâmetros de transporte líquido para as esferas a base de alginato.

\begin{tabular}{lcccc}
\hline & $\begin{array}{c}\text { Raio } \\
\left(\mathbf{1 0}^{-3} \mathbf{m}\right)\end{array}$ & $\begin{array}{c}\theta \\
(\mathbf{1 0} / \mathbf{s})\end{array}$ & $\begin{array}{c}\mathbf{D}_{\mathbf{v}}\left(\mathbf{m}^{2} / \mathbf{s}\right) \\
\left(\mathbf{x ~ 1 0} \mathbf{1 0}^{-10}\right)\end{array}$ & $\mathbf{Q}$ \\
\hline ALG & 0,520 & 0,055 & 0,71 & 0,597 \\
ALG:Cz 1:1 & 0,705 & 0,124 & 6,7 & 0,857 \\
ALG-GC & 0,415 & 0,349 & 18 & 10,6 \\
ALG-GC:Cz 1:1 & 0,415 & 0,580 & 50 & 12,7 \\
\hline
\end{tabular}

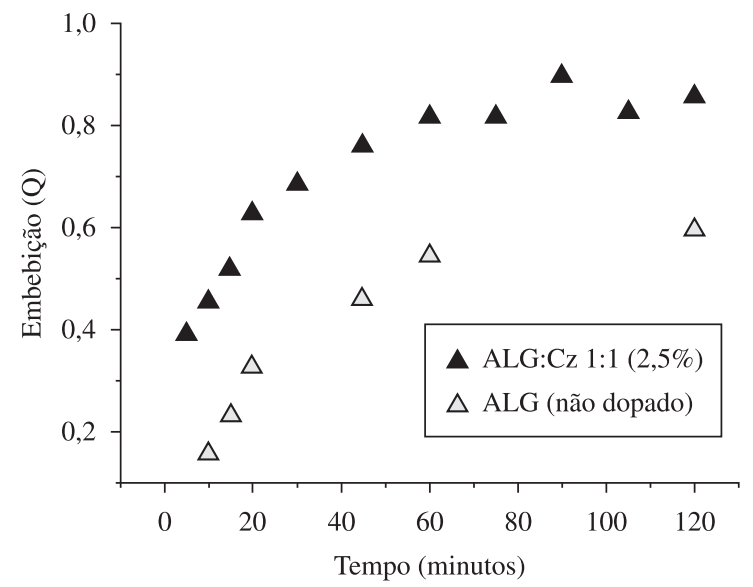

(a) esferas de ALG. Já as esferas revestidas com GC não-dopadas e dopadas com $\mathrm{Cz}$ apresentam valores mais elevados de coeficiente de difusividade, respectivamente de $18 \times 10^{-10} \mathrm{e}$ $50 \times 10^{-10} \mathrm{~m}^{2} / \mathrm{s}$. Desta forma, observa-se que o revestimento de GC pode interferir no mecanismo de formação do "egg-box", levando a uma matriz com menor densidade de reticulação e conseqüentemente maior difusividade e capacidade de embebição. Valores semelhantes de coeficiente de difusividade foram encontrados por Soppirnath e colaboradores $^{[29]}$, cujo diâmetro das esferas de goma guar graftizada com poliacrilamida situou-se na faixa de 0,39 a $0,59 \mathrm{~mm}$ e o coeficiente de difusividade variou de $8,83 \times 10^{-10}$ a $54,43 \times 10^{-10} \mathrm{~m}^{2} / \mathrm{s}$. No entanto, esferas de derivado de celulose apresentaram coeficiente de difusividade mais elevado, com valores na faixa de 0,7 a $7,7 \times 10^{-7} \mathrm{~m}^{2} / \mathrm{s}^{[25]}$.

Foi avaliada a embebição nas esferas cujas formulações apresentaram melhor dopagem e maior eficiência de encapsulamento e comparou-se com a embebição das esferas correspondentes não-dopadas, conforme ilustrado na Figura 3.

As cinéticas de embebição revelam que as esferas não-dopadas apresentam um menor grau de embebição do que as esferas dopadas, provavelmente devido ao menor espaçamento da rede polimérica quando da formação da emulsão, uma vez que a ausência do óleo essencial Cz resulta em uma maior compactação das cadeias do alginato pelo cloreto de cálcio.

\section{Cinética de liberação in vitro}

O estudo da cinética de liberação do óleo $\mathrm{Cz}$ encapsulado nas esferas de ALG e ALG-GC, permite observar-se as eventuais diferenças entre os perfis de liberação, devido a modificação da natureza da matriz polimérica. A Figura 4 mostra a fração de $\mathrm{Cz}$ liberado em função do tempo, para as esferas de ALG e ALG-GC. Observa-se que as esferas ALG-GC:Cz 1:1 apresentaram um perfil de liberação mais prolongado, em função da reticulação iônica das cadeias,

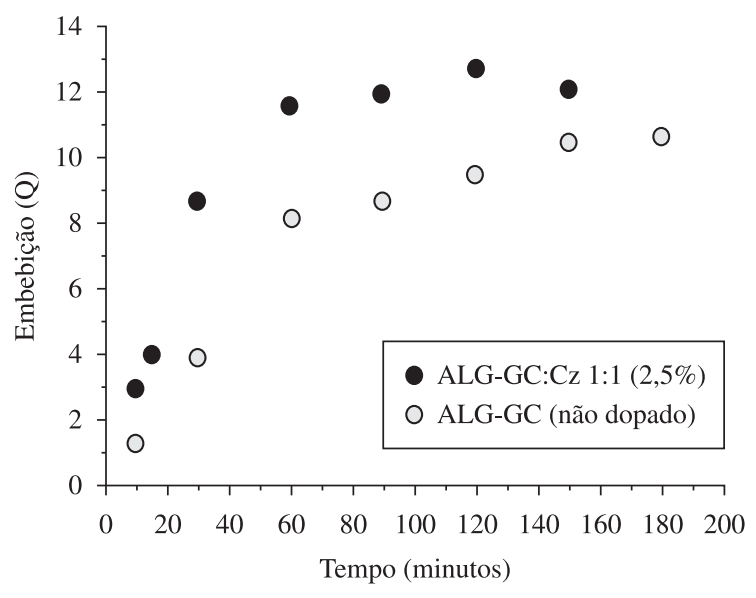

(b)

Figura 3. Embebição das esferas dopadas e não-dopadas de ALG (a) e de ALG-GC (b). 
com $25 \%$ de liberação de óleo após 2 horas, enquanto que as esferas de ALG:Cz liberaram 57\% no mesmo período. Após 4 horas de teste, as esferas ALG e ALG:GC atingiram um patamar, liberando respectivamente 57 e $32 \%$ de $\mathrm{Cz}$ neste período. Isto pode ser atribuído a retenção parcial de óleo na porção hidrofóbica de micelas formadas durante o processo de formação das esferas. A reação de complexação do ALG com os íons cálcio é rápida, levando a formação de estrutura tipo egg-box ${ }^{[30]}$. Desta forma, algumas micelas podem ter ficado retidas dentro da estrutura, dificultando a liberação do óleo em meio aquoso.

A liberação de princípios ativos contidos em matrizes poliméricas embebidas é governada ${ }^{[31]}$ pela Equação 5.

$$
\frac{\mathrm{M}_{\mathrm{t}}}{\mathrm{M}_{\infty}}=\mathrm{Kt}^{\mathrm{n}}
$$

Nesta equação $M_{t} / M_{\infty}$ denota a fração de óleo larvicida liberada, $t$ é o tempo de liberação e $k$ representa uma constante característica do sistema. O coeficiente difusional (n) é um indicativo do mecanismo de liberação. A Tabela 4 mostra os valores de $\mathrm{n}$ e $\mathrm{K}$ obtidos, bem como o coeficiente de correlação da cinética de liberação de óleo essencial para as esferas de ALG:Cz e ALG-GC:Cz.

Para percentuais de liberação de óleo $\leq 50 \%$, tem-se $n$ igual a 0,51 e 0,59 para as esferas de ALG:Cz e ALG-GC:Cz, respectivamente, o que caracteriza um transporte fickiano, ou seja, as velocidades de difusão do principio ativo e de relaxamento das cadeias do polímero são equivalentes ${ }^{[30]}$. Valores semelhantes foram encontrados no estudo da cinética de liberação de fármacos modelo de esferas de ALG, onde o $n$ foi igual a 0,5 indicando que a liberação é governada por processos difusionais ${ }^{[5]}$. Estudos realizados com esferas de alginato reticuladas com glutaraldeido apresentaram valores superiores a 0,6; Kulkarni et al. ${ }^{[12]}$ encontraram valores de $n$ na faixa de 0,74 a 0,94 , enquanto que Soppirnarth e Aminavhabi ${ }^{[29]}$ encontraram uma relação direta entre 0

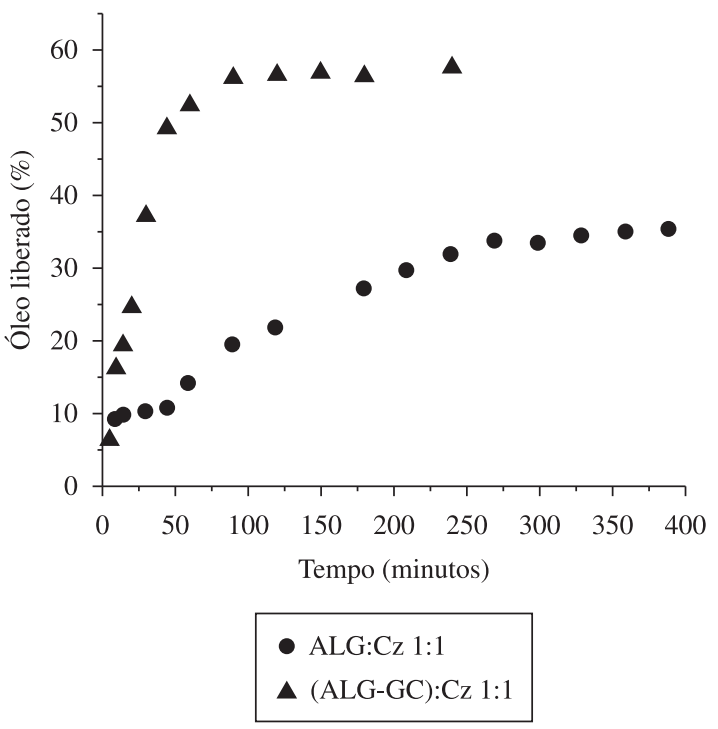

Figura 4. Cinética de liberação das esferas. aumento do coeficiente difusional $n$ de 0,46 para 0,78 com o aumento da quantidade de glutaraldeído na formulação de esferas de goma guar grafitizada com poliacrilamida. Desta forma, pode-se afirmar baseados nestes resultados que o revestimento de GC nas esferas de ALG seguido pela reticulação com glutaraldeído modifica o mecanismo de transporte de fickiano para não-fickiano.

\section{Taxa de mortalidade larval}

Beads de ALG:Cz 1:1 e de ALG-GC:Cz 1:1 foram testadas usando massas de 10 a $50 \mathrm{mg}$ de esferas para o ensaio quanto à mortalidade larval, conforme pode ser visto na Figura 5. Observa-se que para uma mesma quantidade de esferas dopadas $(50 \mathrm{mg}$ de esferas, correspondente a uma concentração de 85 ppm de óleo), as diferentes formulações apresentam índices de mortalidade larval diferentes, na faixa de 78 a 100\%, após 24 horas. Comparando com o valor do $\mathrm{LD}_{50}$ da literatura do $\mathrm{Cz}$ que é de $28 \mathrm{ppm}$, observa-se o princípio ativo encapsulado nas esferas mantém o caráter larvicida e atinge índices de mortalidade coerentes com o obtido para o óleo puro. Na dosagem de 30 e $20 \mathrm{mg}$ de esferas, que corresponde a concentrações respectivas na faixa de 50 e $36 \mathrm{ppm}$ de $\mathrm{Cz}$, as esferas de ALG apresentaram um valor médio de 95 e $65 \%$ de mortalidade, respectivamente, enquanto que as esferas de ALG-GC apresentaram valores menores (48 e $28 \%$ ). No entanto, para as esferas de ALG-GC:Cz a taxa de mortalidade aumentou progressivamente em 48 horas de ensaio, atingindo valores de 60 a $98 \%$, similares aos apresentados pelas esferas ALG:Cz. Desta forma, observa-se que o perfil de liberação do óleo é mais controlado para as esferas ALG-GC:Cz, onde a mortalidade larval ocorre de

Tabela 4. Parâmetros da cinética de liberação in vitro de $\mathrm{Cz}$ pelas esferas de ALG e ALG-GC.

\begin{tabular}{lccc}
\hline \multicolumn{1}{c}{ Esferas } & $K$ & $n$ & $\mathbf{R}^{2}$ \\
\hline ALG:Cz 1:1 & 0,072 & 0,51 & 0,987 \\
ALG-GC:Cz 1:1 & 0,053 & 0,59 & 0,992 \\
\hline
\end{tabular}

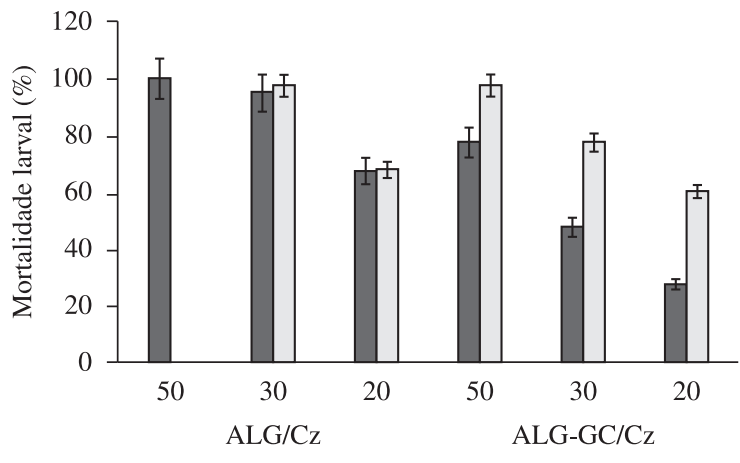

Massa de esferas (mg)

\section{4 horas}

48 horas

Figura 5. Mortalidade Larval em função da quantidade de amostra para as esferas ALG:Cz 1:1 e ALG-GC:Cz 1:1. 
forma progressiva. Tal fato pode ser atribuído a reticulação das cadeias dos polímeros com glutaraldeído, o que confere um maior grau de empacotamento e conseqüentemente um perfil de liberação mais controlado, permitindo uma taxa de mortalidade mais prolongada.

A estabilidade do sistema polimérico foi investigada através da determinação da dopagem das esferas após 2,8 , 16, 30 e 64 dias de preparação. Conforme pode ser visto na Figura 6, as esferas são eficientes para retenção do óleo essencial, havendo uma perda de apenas $35 \%$ em 70 dias.

\section{Análise térmica}

As esferas dopadas ALG:Cz 1:1 e ALG-GC:Cz 1:1 e as de ALG não-dopadas foram avaliadas por análise termogravimétrica, conforme ilustrado na Figura 7a. Os dados de perda de massa e as temperaturas de pico para o eventos térmicos estão sumarizados na Tabela 5. Observa-se a existência de cinco principais eventos térmicos para as esferas de ALG. Popa e colaboradores ${ }^{[30]}$, reportaram que microesferas de alginato reticuladas com cálcio apresentaram a decomposição em três etapas, com o primeiro evento atribuído a evaporação de água, o segundo devido a formação

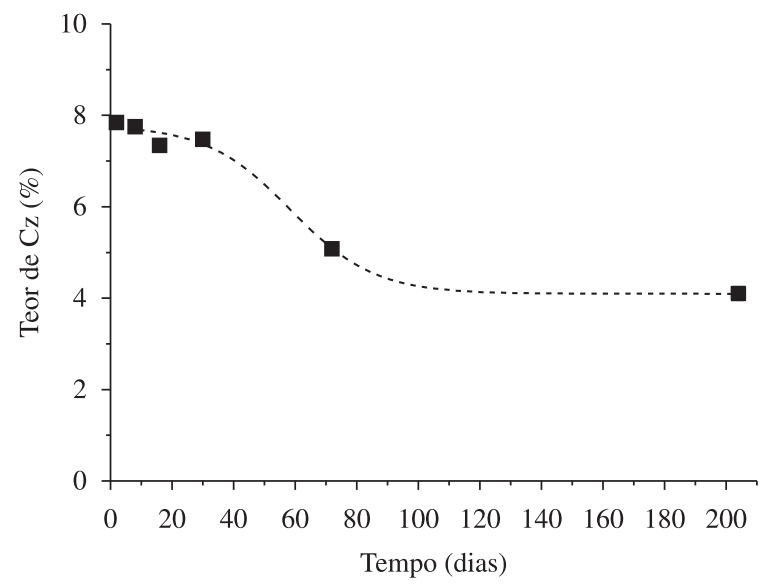

Figura 6. Estabilidade das esferas ALG:Cz 1:1.

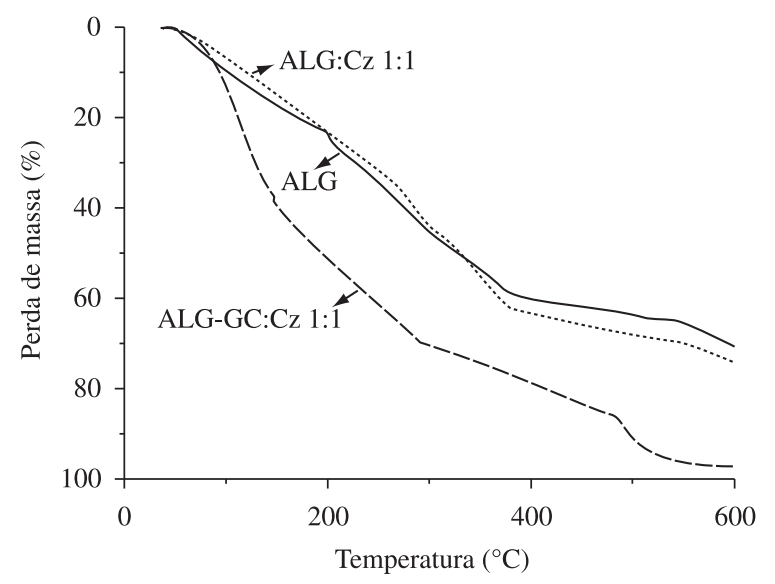

(a) de carbonato de sódio e o terceiro devido a carbonização das cadeias poliméricas. Desta forma, pode-se afirmar que a decomposição das esferas de ALG e de ALG-GC apresentam eventos térmicos em faixas de temperaturas de decomposição similares, com a evaporação da água ocorrendo na faixa

Tabela 5. Eventos térmicos da análise de TGA das esferas de ALG e ALG-GC.

\begin{tabular}{|c|c|c|c|}
\hline Amostra & Eventos & $\begin{array}{l}\text { Temperatura } \\
\text { de pico }\left({ }^{\circ} \mathbf{C}\right)\end{array}$ & $\begin{array}{c}\text { Perda de massa } \\
(\%)\end{array}$ \\
\hline \multirow{5}{*}{ ALG } & $1^{\mathrm{o}}$ & 85 & 22,29 \\
\hline & $2^{\circ}$ & 209 & 16,08 \\
\hline & $3^{\circ}$ & 289 & 13,57 \\
\hline & $4^{\circ}$ & 368 & 5,76 \\
\hline & $5^{\circ}$ & 572 & 5,61 \\
\hline \multirow{5}{*}{ ALG:Cz 1:1 } & $1^{\mathrm{o}}$ & 132 & 13,31 \\
\hline & $2^{o}$ & 209 & 17,12 \\
\hline & $3^{\circ}$ & 293 & 14,61 \\
\hline & $4^{\circ}$ & 360 & 9,45 \\
\hline & $5^{\circ}$ & 572 & 5,52 \\
\hline \multirow{5}{*}{$\begin{array}{c}\text { ALG-GC:Cz } \\
1: 1\end{array}$} & $1^{\mathrm{o}}$ & 102 & 36,2 \\
\hline & $2^{o}$ & 208 & 27,8 \\
\hline & $3^{\circ}$ & 343 & 7,2 \\
\hline & $4^{\circ}$ & 440 & 10,1 \\
\hline & $5^{\circ}$ & 550 & 14,9 \\
\hline
\end{tabular}

Tabela 6. Eventos térmicos da análise de Calorimetria Diferencial de Varredura (DSC) das esferas de ALG e ALG-GC.

\begin{tabular}{ccccc}
\hline \multirow{2}{*}{ Esferas } & \multicolumn{2}{c}{ Exotérmico } & \multicolumn{2}{c}{ Endotérmico } \\
\cline { 2 - 5 } & $\begin{array}{c}\text { Temperatura } \\
\left({ }^{\mathbf{C}} \mathbf{C}\right)\end{array}$ & $\begin{array}{c}\Delta \mathbf{H} \\
\left(\mathbf{m J . g}^{-1}\right)\end{array}$ & $\begin{array}{c}\text { Temperatura } \\
\left({ }^{\mathbf{C}} \mathbf{C}\right)\end{array}$ & $\begin{array}{c}\Delta \mathbf{H} \\
\left(\mathbf{m J . g}^{-1}\right)\end{array}$ \\
\hline \multirow{2}{*}{ ALG } & 367 & $-2,86$ & 84 & 2,19 \\
& & & 195 & 2,46 \\
ALG:Cz 1:1 & 375 & $-4,65$ & 135 & 16,00 \\
ALG-GC:Cz & 316 & $-0,77$ & 68 & 2,22 \\
1:1 & 406 & $-0,31$ & 217 & 0,31 \\
\hline
\end{tabular}

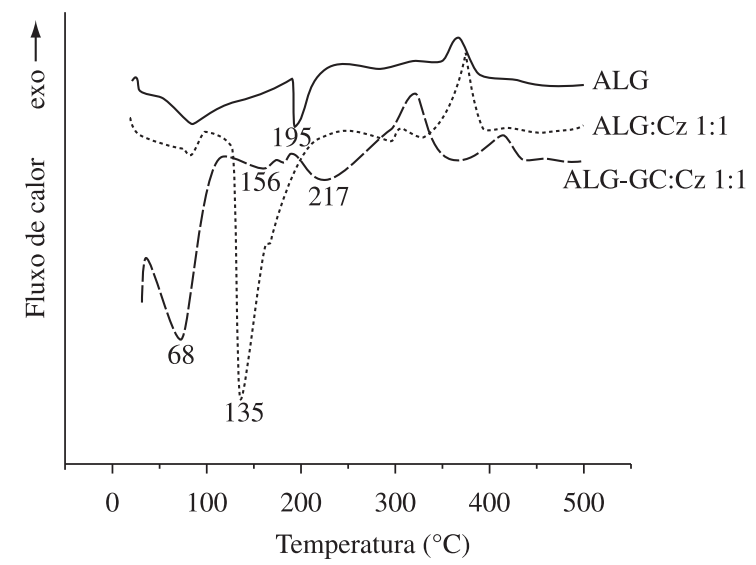

(b)

Figura 7. a) Análise termogravimétrica (TGA) e b) Análise por Calorimetria de Varredura Diferencial (DSC) das esferas de ALG não dopadas, ALG:Cz 1:1 e ALG-GC:Cz 1:1. 
de $85-132{ }^{\circ} \mathrm{C}$, e a formação de carbonatos em $209{ }^{\circ} \mathrm{C}$ e a carbonização das cadeias poliméricas no intervalo de 297 a $576^{\circ} \mathrm{C}$.

Na Figura 7b e Tabela 6, os dados de DSC mostram que as esferas de ALG não dopadas apresentam um pico endotérmico referente a fusão na temperatura de $194{ }^{\circ} \mathrm{C}$, consistente com o reportado em outros trabalhos ${ }^{[32-34]}$. As esferas ALG:Cz apresentaram um deslocamento da temperatura de fusão para $140{ }^{\circ} \mathrm{C}$, devido possivelmente a interação da matriz com o óleo encapsulado. As esferas de ALG-GC:Cz apresentam um pico endotérmico referente a perda de água a $65{ }^{\circ} \mathrm{C}$, característico da goma de cajueiro, bem como as transições endotérmicas nas temperaturas de 156 e $219^{\circ} \mathrm{C}$, referentes à fusão do ALG. A exibição de duas temperaturas de fusão para o alginato foi também observada no trabalho de Patel e colaboradore $^{[33]}$, sendo um indicativo de dispersão molecular do principio ativo dentro da matriz. Embora a incorporação de $\mathrm{Cz}$ nas esferas diminui a temperatura de fusão, ocorre o aumento da temperatura de degradação, tornando o sistema mais estável, conforme pode ser visto a partir dos dados das amostras ALG e ALG:Cz 1:1. Já a adição de GC (ALG-GC:Cz 1:1) resulta em fusões à temperaturas mais elevadas, com temperatura de decomposição de ALG mais baixa. GC decompõe-se a $406^{\circ} \mathrm{C}$, valor mais elevado do que reportado por Silva et al. ${ }^{[35]}$

\section{Conclusões}

Óleo essencial de Croton zehntneri Pax et Hoffm foi encapsulado de forma satisfatória nas esferas de ALG e ALG-GC, com teores de dopagem entre 1,2 e 10,7\%, sendo que a eficiência da matriz polimérica na preservação do principio ativo foi comprovada através da presença de quantidade significativa de óleo na matriz mesmo após cerca de 70 dias. A análise morfológica revelou uma regularidade esférica, com superfície porosa. A presença do óleo essencial nas esferas foi comprovada ainda por meio da análise de espectroscopia de infravermelho. A concentração ideal de ALG, que maximiza a dopagem e o grau de encapsulamento é de 2,5\%, para as amostras ALG:Cz 1:1 e ALG-GC: $\mathrm{Cz}$ 1:1. A adição da GC e a reticulação das esferas não altera a dopagem mas modifica o caráter hidrofílico da matriz polimérica, aumentando significativamente o valor do coeficiente de difusividade e do grau de embebição Q. A analise térmica revelou que a incorporação de $\mathrm{Cz}$ e GC resultam em uma matriz polimérica mais estável, com clara evidencia do caráter interativo das cadeias poliméricas envolvidas. $\mathrm{O}$ perfil de liberação in vitro mostrou-se com efeito mais prolongado para a amostra recorberta com GC, ALG-GC:Cz 1:1, devido a reticulação de suas cadeias. As esferas revelaram-se efetivas para a liberação do larvicida, com mortalidade larval na faixa de 72 a $100 \%$, se constituindo em um sistema eficiente para encapsulamento e liberação de larvicida, representado assim uma alternativa no combate a dengue.

\section{Agradecimentos}

Ao CNPQ, pela bolsa PIBIC-UFC; à REDE NANOGLICOBIOTEC/CNPQ pelo auxilio financeiro para execução das pesquisas e à técnica química Laíse Costa Borba pela execução do MEV.

\section{Referências Bibliográficas}

1. Hejazi, R. \& Amiji, M. - Pharmaceut. Dev. Tech., 8, p. 253 (2003).

2. Hamidi, M.; Azadi, A. \& Rafiei, P. - Adv. Drug Deliv. Rev., 60, p.1638 (2008).

3. Pasparakis, G. \& Bouropoulos, N. - Int. J. Pharm., 323, p. 34 (2006).

4. Sarmento, B.; Ribeiro, A.; Veiga, F.; Sampaio, P.; Neufeld, R. \& Ferreira, D. - Pharm. Res., 24, p. 12 (2007).

5. Mladenovska, K.; Cruaud, O.; Richommed, P.; Belamie, E.; Raicki, R. S.; Venier-Julienne, M. C.; Popovski, E.; Benoit, J. P. \& Goracinova, K. - Int. J. Pharm., 345, p. 59 (2007).

6. Tu, J.; Bolla, S.; Barr, J.; Miedema, J.; Li, X. \& Jasti, B. - Int. J. Pharm., 303, p. 171 (2005).

7. Cordoba A.; Cuellar, N.; Gonzalez, M. \& Medina, J. Carbohydr. Polymer., 73, p. 409 (2008).

8. Salmieri, S. \& Lacroix, M. - J. Agric. Food Chem., 54, p. 10205 (2006).

9. Kulkarni, A. R.; Soppimath, K. S.; Aminabhavi, T. M. \& Rudzinski, W. E. - Eur. J. Pharm. Biopharm., 51, p. 127 (2001).

10. Anal, A. K. \& Stevens, W. F. - Int. J. Pharm., 290, p. 45 (2005).

11. Kulkarni, A. R.; Soppimath, K. S.; Aminabhavi, T. M. \& Dave, A. M. - J. Appl. Polym. Sci., 85, p. 911 (2002).

12. Kulkarni, A. R.; Soppimath, K. S.; Aminabhavi, T. M.; Dave A. M. \& Mehta, M. H. - J. Control. Release, 63, p. 97 (2000).

13. Inoue, M.; Ontsubo, T.; Imai, M. - in: Proceedings of the 24 International Symposium on Controlled Release ofBioactive Materials, p. 733, Sweden, june (1997).

14. Isiklan, N. - J. Appl. Polymer Sci., 99, p. 1310 (2006).

15. Paula, H. C. B.; Gomes, F. J S. \& Paula, R. C. M. Carbohydr. Polymer., 48, p. 313 (2002).

16. Paula, H. C. B.; Peixoto, M. C. P. \& Nascimento, R. F. - in: Anais do 5 International Symposium on Natural Polymers and Composites, São Paulo-SP, (2004).

17. Maciel, J. S.; Paula, H. C. B.; Miranda, M. A. R.; Sasaki, J. M. \& Paula, R. C. M. - J. Appl. Polymer Sci., 99, p. 326 (2006). 
18. Paula, R. C. M.; Heatley, F. \& Budd, P. M. - Polymer Int., 45, p. 54 (1998).

19. Morais, S. M.; Catunda Júnior, F. E. A.; Silva, A. R. A.; Stone, J.; Neto, M.; Rondina, D. \& Cardoso, J. H. L. Quim. Nova, 29, p. 907 (2006).

20. Camurça-Vasconcelos, A. L. F.; Bevilaqua, C. M. L.; Morais, S. M.; Maciel, M. V.; Costa, C. T. C.; Macedo, I. T. F.; Oliveira, L. M. B.; Braga, R. R.; Silva, R. A. \&Vieira, L. S. - Vet. Parasitol., 148, p. 288 (2007).

21. Morais, S. M.; Cavalcanti, E. S. B.; Bertini, L. M.; Oliveira, C. L. L.; Rodrigues, J. R. B. \& Cardoso, J. H. L. - J. Am. Mosq. Control Assoc., 22, p. 161 (2006).

22. Stadler, A. S; Ferreira, T. B. R. C.; Ribeiro, W. L. C. R.; Paula, H. C. B. - in: Anais da Mostra Internacional de Ciência e Tecnologia, p. 473, Novo Hamburgo - RS. (2008).

23. Lima, L. P.; Paula, H. C. B.; Frota, M. M. A. \& Ribeiro, W. L. C. - in: Anais da Expo-Ciencia Internacional, Fortaleza - CE. (2004).

24. Thangam, T. S. \& Katiesan, K. - Botanic Materials, 34, p. 433 (1991).

25. El-Bahri, Z. \& Taverdet, J. L. - Polymer Bull., 59, p. 709 (2007).

26. Lawrie, G.; Keen, I.; Drew, B.; Chandler-Temple, A.; Rintoul L.; Fredericks, P. \& Grøndahl, L. Biomacromolecules, 8, p. 2533 (2007).
27. Freire, R. S.; Morais, S. M.; Catunda Junior, F. E. A. \& Pinheiro, D. C. S. N. - Bioorg. Med. Chem., 13 p. 4353 (2005).

28. Paula, H. C. B.; Paula R. C. M. \& Bezerra, S. K. F. J. Applied Polym. Sci., 102, p. 395 (2006).

29. Soppirnath, K. S. \& Aminabhavi, T. M. - Eur. J. Pharm. Biopharm., 53, p. 87 (2002).

30. Velings, N. M. \& Mestdagh, M. M. - Polym. Gels Networks, 3 p. 31 (1995).

31. Korsmeyer, R. W. \& Pepas, N. A. - J. Memb. Sci., 9, p. 211 (1981).

32. Mandal, S.; Basu S. K. \& Sa, B. - AAPS PharmSciTech, 10, p. 1348 (2009).

33. Patel Y. L.; Sher, P. \& Pawar, A. P. - AAPS PharmSciTech, 7, p. E1 (2006).

34. Rahman, Z.; Kohli, K.; Khar, R. K.; Ali, M.; Charoo, N. A. \& Shamsher, A. A. A. - AAPS PharmSciTech, 7 (2006).

35. Silva D. A.; Feitosa, J. P. A.; Maciel, J. S.; Paula, H. C. B. \& Paula, R. C. M. - Carbohydr. Polymer., 66, p. 16 (2006).

Enviado: 31/10/09

Reenviado: 21/12/09

Aceito: $19 / 01 / 10$

DOI: $10.1590 / \mathrm{S} 0104-14282010005000019$ 